\title{
Benefits of psychodynamic group therapy on depression, burden and quality of life of family caregivers to Alzheimer's disease patients
}

\author{
Dorli Kamkhagi ${ }^{1}$, Ana Carolina Oliveira Costa ${ }^{1}$, Sandra Kusminsky¹, Deborah Supino1, Breno Satler Diniz², \\ Wagner Farid GattaZ ${ }^{1}$, Orestes Vicente ForlenZa ${ }^{1}$
}

1 Laboratory of Neuroscience (LIM-27), Department and Institute of Psychiatry, Faculty of Medicine, University of Sao Paulo (USP), Sao Paulo, SP, Brazil.

2 Department of Mental Health, Faculty of Medicine, Federal University of Minas Gerais (UFMG), Belo Horizonte, MG, Brazil.

Received: 10/27/2015 - Accepted: 11/9/2015

DOl: 10.1590/0101-60830000000067

\begin{abstract}
Background: Family members providing continuous care to demented patients suffer from severe burden that impairs quality of life and often evolves to depression. Objective: This study aims to evaluate the effect of psychodynamic group psychotherapy (PGT) compared to body awareness therapy (BAT) on caregiver burden, depressive symptoms, and quality of life among family caregivers to Alzheimer disease (AD) patients. Methods: Thirty-seven healthy family caregivers were randomly allocated to receive PGT $(n=20)$ or BAT $(n=17)$. Interventions were administered in the format of 14 weekly group sessions. Outcome measures were: modification of scores on Zarit Burden Scale, Beck Depression Inventory and WHO-QoL Scale. Results: Participants in the PGT group displayed significant reduction on burden $(\mathrm{p}=0.01)$ and depression scores $(\mathrm{p}=0.005)$, and improved quality of life $(\mathrm{p}=0.002)$, whereas those in the BAT group showed improvements in burden of care $(\mathrm{p}=0.001)$ and quality of life $(\mathrm{p}=0.01)$, but not on depressive symptoms $(\mathrm{p}=0.13)$. Discussion: Psychodynamic psychotherapy was associated with amelioration of depressive symptoms, but overall benefits on burden of care and quality of life were similar irrespective of the type of intervention, i.e., psychologically-oriented or not. We hypothesize that these interventions can be complementary to improve depression and burden of care among family caregivers of AD patients.
\end{abstract}

Kamkhagi D et al. / Arch Clin Psychiatry. 2015;42(6):157-60

Keywords: dementia, Alzheimer's disease, caregiver, burden, depression, quality of life, psychodynamic therapy, body awareness therapy.

\section{Introduction}

As a consequence of the increasing prevalence of Alzheimer disease (AD) worldwide in the last decades, the number of family caregivers has also increased steadily, particularly in settings where the access to professional caregiving is limited by financial or cultural reasons. This role is well accepted to bear a high-risk for the development of physical and mental health impairments, which are indeed observed in a high proportion of caregivers. This is largely explained by the increased burden generated by the excess of work and responsibilities involved in caring for demented patients, in addition to the emotional implications of having a family member with declining cognitive skills and functional autonomy, in addition to an array of behavioral problems. Therefore, consequences to global health of family caregivers involves physical and psychological symptoms, which in due term may overburden the public healthcare system 1 .

There are two basic types of providers of care to dementia patients, i.e., professional and family caregivers, the latter being much more prone to experience symptoms of stress and burden, both physically and emotionally. The role of the professional caregiver subsumes previous training on this particular task, which is regarded as a profession with technical specificities. Therefore, in addition to being paid for, professional caregivers perform their activity on shifts and are entitled to hours of rest and vacations. On the other hand, family caregivers usually have to incorporate this new and highly demanding activity in their routine, without any previous warning or technical preparation ${ }^{2}$. Frequently, this person has another occupation or profession, which he or she may have to abdicate or cutback.

The impact suffered by caregivers to dementia patients seems to be higher than that observed when providing care for patients with physical health problems ${ }^{3}$. In $\mathrm{AD}$, along with disease progression, cognitive and functional deterioration will cause further loss of autonomy and increased dependence to perform basic activities of the daily living. As a consequence of that, caregivers will have not only to dedicate more time caring for the patient, but also perform this activity under more stress ${ }^{4}$. Caregiver burden therefore arises not only from the psychological distress associated with this role, but also from chronic fatigue, derived from long hours of assistance to the family member, seldom without periods of rest and adequate sleep ${ }^{5}$. The nature and quality of the previous relationship that the caregiver has endured with the patient through their previous lives are also determinants of the success of the adaptation to the caregiver's role 6 . In addition, these coping abilities are critically linked to the caregiver's personality and emotional state, which are decisive to determine how he or she will deal with the multiple demands that arise in the long and deteriorating course of dementia, in the completion of a role that in not always valued as it should be by other family members. The sum of all these factors is likely to generate anxiety, emotional distress and ultimately depression, which can further impair the caregiver's capacity to accurately assist the patient with dementia. The preservation of physical and mental health of family caregivers to dementia patients must be understood as an asset to the efficient and ethical fulfillment of this important role ${ }^{7-9}$. There is an estimate of $46 \%$ of increase in the demand for healthcare services for $\mathrm{AD}$ patient caregivers ${ }^{10}$. In addition, up to $60 \%$ of family caregivers to AD patients may develop physical and/or psychological symptoms, including hypertension, digestive problems, sleep disorders, respiratory diseases, immunologic system deficiency, depression, anxiety and insomnia ${ }^{7,11}$

The primary objective of the present study was to assess the benefits of a psychologically oriented intervention (psychodynamic group psychotherapy, PGT) compared to a non-psychologically oriented intervention (body awareness therapy, BAT) for the treatment of caregiver burden among family caregivers of patients with AD. Our secondary objective was to evaluate the effects of these interventions on depressive symptoms and quality of life of these subjects. To the best of our knowledge, there are no controlled studies assessing the impact of the psychodynamic group psychotherapy on depressive symptomatology and burden of care among family caregivers to demented $\mathrm{AD}$ patients. 


\section{Methods}

\section{Participants and study design}

The study was conducted at the psychogeriatric clinic of a tertiary hospital in Brazil (Institute of Psychiatry, Faculty of Medicine, University of Sao Paulo) between February and December 2013. Participants were recruited from the hospital catchment area and with the aid of media advertisements. Inclusion criteria were: being a spouse or first-degree relative to a non-institutionalized demented patient with mild or moderate AD; dwelling in the same household as the patient; providing care for at least four hours a day. Exclusion criteria were: impediment or unwillingness to participate in all procedures of the study (baseline and endpoint screening plus intervention); having a psychiatric diagnosis that might preclude group work; current or previous participation (in the previous six months) in any psychoeducational program or $\mathrm{AD}$ support group; being less than 35 years old. Thirty-seven cognitively healthy family caregivers were enrolled to the study. All participants signed the informed consent. The study was approved by the local Ethics Committee and conducted under the tenets of the Helsinki declaration.

The study was designed to be a single-blinded, controlled intervention trial, in which the effect of a psychological intervention is compared to a non-psychologically oriented intervention addressing caregiver burden, depressive symptoms and quality of live in a sample of family caregivers to AD patients. After the baseline screening assessment, in which participants underwent evaluation of physical capacity and balance, family caregivers were randomly allocated to intervention groups. Table 1 presents social-demographic and clinical characteristics of the sample. Subjects in the intervention group $(\mathrm{n}=$ 20) received psychodynamic group psychotherapy (Group 1, PGT) and subjects in the comparison group $(n=17)$ received body awareness therapy (Group 2, BAT). Both interventions were administered once a week in a total of 14 group sessions of 90 minutes of duration each. PGT sessions were administered by two experienced psychologists. The facilitators of BAT sessions were a physical therapist and a psychologist. Outcome measures were the modification (endpoint vs. baseline) of scores on the Zarit Burden Scale ${ }^{12}$, Beck Depression Inventory ${ }^{13}$, and WHO-QoL Scale ${ }^{14}$. Participant in Group 2 were additionally assessed with the Body Awareness Questionnaire ${ }^{15}$. The Clinical Dementia Rating Scale (CDR) ${ }^{16}$ was administered to ascertain the degree of impairment of $\mathrm{AD}$ patients who were cared for by their relatives.

Table 1. Demographic data of participants in both groups

\begin{tabular}{|l|c|c|c|}
\hline \multirow{2}{*}{} & \multicolumn{2}{|c|}{ Group } & \multirow{2}{*}{ p-value } \\
\cline { 2 - 3 } & PGT & BAT & \\
\hline Gender & $25 \%$ & $30 \%$ & 0.7 \\
Men & $75 \%$ & $70 \%$ & \\
Woman & $62.1 \pm 9.9$ & $55.7 \pm 14.9$ & 0.1 \\
\hline Age (years) & $15 \%$ & $6 \%$ & 0.3 \\
\hline Education & $85 \%$ & $94 \%$ & \\
Elementary school & & \\
High school or higher & &
\end{tabular}

PGT: Psychodynamic group therapy; BAT: Body awareness therapy.

\section{Interventions}

In the PGT group, several aspects of the caregiver roles were dealt with through the intervention program, reinforcing their resilience abilities. The most frequent aspects, usually raised spontaneously by participants (otherwise prompted by facilitators) were: loneliness and helplessness associated with the caregiver's role, occurrence of family conflicts, and changing roles within the family dynamics. Participants were encouraged by the facilitators to get in contact with their feelings of sadness, frustration, anger and guilt, and to further name and elaborate the feelings they experience when dealing with these situations. In the first moment, the participants were asked to think about their previous relationship with the patient under their care, as it used to be before the onset of dementia, and then to update this notion to the present reality, thus enabling the elaboration of new ways to maintain an affectionate caring relationship with their relative. In a second moment, facilitators of the PGT group worked on other roles that the caregiver used to play in the family dynamics before the onset of the illness, and the necessary adaptations to the present time, particularly due to the necessity of performing unwanted roles and having to abandon (or at least cutback) the engagement in other competing roles. Some effort is dedicated by facilitators to establish and reinforce empathy with the difficulties faced by the subject, discussing strategies to better cope with the various sources of burden of care. Towards the completion of the program, facilitators discuss the lack of recognition of the caregiver's efforts by other family members and encourage participants to nominate their perceived and unattended rights; this "caregiver declaration of rights" usually warrants them a place of existence and gratefulness for their feelings and concerns.

The BAT intervention was designed according to the "Functional Symbolic Dynamics" methodology, created by Marcia Taques Bittencourt ${ }^{17}$, which has as a major goal to bring the patient to bodily self-awareness. This intervention started with a physical evaluation by the therapist and uses several physiotherapeutic backgrounds such as calatonia, eutonia, feldenkrais, relaxing, somatic experience, spontaneous movements and dance. Petho Sándor ${ }^{18}$ established in 1974 the basis for the utilization of soft touches in specific points, which uses the high potential of the skin sensitivity, promoting a multi-sensorial experience, physical and psychological rebalancing, and leading the recipients of the intervention to pay more attention to themselves. Body relaxation is considered a psychophysiological reconditioning method. In eutonia, through attention and self-observation, using auxiliary objects, the individual can get in contact with his/her internal rhythm. The sequence of Feldenkrais slow movements will explore the patterns and limits of the body. During the sessions, some tools were used to awaken new perceptions, such as: bed linen, clay, little balls and drawings.

\section{Statistical analysis}

Student's t test and Chi-squared tests were used to evaluate differences between intervention groups on socio-demographic and clinical variables in the initial evaluation (i.e. pre-intervention). The efficacy of the interventions on the measures of primary and secondary measures was evaluated through repeated measure analysis of variance (ANOVA) using the pre and post-intervention scores in the outcome measures. All statistical analyses were done with the SPSS software (USA).

\section{Results}

There were no significant differences on socio-demographic and clinical variables between subjects in the PGT and the BAT (Table 1).

Repeated measure analysis of variance showed that there was a significant improvement in depressive symptoms in the whole sample, regardless of the intervention group (within-subject contrast: $\mathrm{F}(1,35)=19.95, \mathrm{p}<0.001$; group ${ }^{\star}$ factor interaction: $\mathrm{F}(1,35)=0.43$, $\mathrm{p}=0.51$ ). In addition, subjects in both groups improved the perceived caregiver burden (within-subject contrast: $\mathrm{F}(1,35)=53.12, \mathrm{p}<0.001$; group ${ }^{\star}$ factor interaction: $\left.f(1,35)=0.03, p=0.87\right)$ and quality of life (within-subject contrast: $\mathrm{F}(1,35)=19.95, \mathrm{p}<0.001$; group ${ }^{\star}$ factor interaction: $f(1,35)=0.43, p=0.51)$, independent of the intervention group. As expected, subjects in the BAT group significantly improved their body self-awareness in contrast to those in the PGT group (within-subject contrast: $\mathrm{F}(1,35)=0.09, \mathrm{p}<0.76$; group ${ }^{\star}$ factor interaction: $\mathrm{f}(1,35)=4.83, \mathrm{p}=0.035)$ (Table 2). 
Table 2. Scores on the outcomes assessment scale before and after intervention

\begin{tabular}{|c|c|c|c|c|}
\hline & \multicolumn{2}{|c|}{ PGT } & \multicolumn{2}{|c|}{ BAT } \\
\hline & Mean & SD & Mean & SD \\
\hline BDI (baseline) & 15.8 & 6.4 & 19.0 & 10.5 \\
\hline BDI (after intervention) 1 & 9.5 & 4.3 & 14.4 & 9.6 \\
\hline ZBS (baseline) & 35.3 & 12.8 & 48.9 & 15.1 \\
\hline ZBS (after intervention)2 & 24.0 & 7.7 & 37.1 & 9.8 \\
\hline WH0-0oL (Baseline) & 88.8 & 13.0 & 76.6 & 14.4 \\
\hline WHO-OoL (after intervention) ${ }^{3}$ & 102.0 & 13.7 & 86.2 & 14.8 \\
\hline BA0 (Baseline) & 32.4 & 7.8 & 31.5 & 6.3 \\
\hline BA0 (after intervention) ${ }^{4}$ & 29.5 & 7.4 & 33.8 & 6.8 \\
\hline
\end{tabular}

PGT: Psychodynamic GROUP THERAPY; BAT: Body Awareness Therapy; BDI: Beck Depression Inventoire; ZBS: Zarit Burden Scale; WHO-Quality of Life Scale; BAQ: Body Awareness Questionnaire. 1 Within-subject contrast: $F(1,35)=19.95, p<0.001$; group $*$ factor interaction: $F(1,35)=0.43, p=0.51$. ${ }^{2}$ Within-subject contrast: $F(1,35)=53.12, p<0.001$; group ${ }^{*}$ factor interaction: $f(1,35)=0.03, p=0.87$. ${ }^{3}$ Within-subject contrast: $F(1,35)=19.95, p<0.001$; group $*$ factor interaction: $F(1,35)=0.43, p=0.51$. ${ }^{4}$ Within-subject contrast: $F(1,35)=0.09, p<0.76$; group*factor interaction: $f(1,35)=4.83, p=0.035$

\section{Discussion}

The major goal of the present study was to determine whether two distinct treatment approaches - one psychologically-oriented and the other intended to provide emotional comfort through body awareness therapy - might help attenuate caregiver burden and related symptoms in a sample of family caregivers to mild- and moderately demented $\mathrm{AD}$ patients. Our results suggest that both interventions were effective reducing the magnitude of caregiver burden and resulted in a better perception of quality of live, but only participants who received psychodynamic group therapy (PGT) experienced a reduction in depressive symptoms. On the other hand, as expected, recipients of body awareness intervention (BAT) had additional improvement in relation to body awareness. Interventions focused on body awareness have been used as therapeutic alternative in several diseases, including fibromyalgia syndrome, targeting both physical and psychological (largely depressive) symptoms ${ }^{19}$. Such interventions have been shown to be effective improving quality of life of these patients ${ }^{20,21}$. To the best of our knowledge, no previous studies published in the literature assessed the efficacy of BAT on caregiver burden.

The multidisciplinary psychogeriatric service in which the present study was conducted is located at a university-based, tertiary hospital that provides healthcare for a substantial catchment area; therefore the high demand for specialized attention to this population reinforces the need to optimize access to treatment through group interventions. In spite of the clinical perception of benefit, there is limited evidence derived from controlled trials of the efficacy of group therapies to attenuate caregiver burden in families of patients with dementia. In the present study, we compared two approaches that have been clinically provided to family caregivers of patients with $\mathrm{AD}$ attending our memory clinic, with relative success over the past years.

Among various types of interventions dedicated to deal with caregiver burden, psycho-education approaches are by far the most studied ones. Benefits have been shown by both individual and group interventions, and recent studies suggested that even remote models of attention provided by telephone or web-based interviews may also be efficacious ${ }^{22}$. In a single-blinded randomized controlled trial with 38 family caregivers of persons with dementia ${ }^{22}$, presented benefits of a telephone-based psycho-educational program delivered through twelve sessions. Compared to subjects in the control group, who were given a DVD containing general information about caregiving, recipients of the intervention reported better self-efficacy and lesser degrees of burden. This approach may be particularly useful to provide support for caregivers living in remote areas devoid of proper health facilities, or for those who cannot attend regular support programs offered by memory clinics.
To date, there is limited evidence of effective interventions for reducing stress related to the burden of care among family caregivers. In a recent study ${ }^{23}$, presented the results from a randomized controlled trial with 38 family caregivers to demented patients addressing the efficacy of a mindfulness-based stress reduction program delivered in eight weeks, as compared to standard social support. Participants in the intervention group reported significantly lower levels of perceived stress and mood abnormalities in addition to long-term improvements on several psychosocial outcomes. In another controlled study conducted in Taiwan ${ }^{24}$, addressed the effectiveness of a psychosocial intervention aiming at improving coping abilities in order to reduce caregiver burden in a sample of 57 caregivers of patients with dementia. Participants in the intervention group received, every two weeks, information on general aspects of dementia, availability of social resources, problem-solving and emotional support, whereas participants in the control group received reinforcements to regular clinical management by telephone contacts. Discontinuation rate was high (19.3\%); nonetheless, the authors concluded that the psychosocial intervention was effective reducing caregiver burden, helping caregivers to adopt better coping strategies.

Acton and Kang ${ }^{25}$ conducted a meta-analysis to address the effect of various interventions on the burden of persons caring for family members with dementia. Among distinct techniques designed to help caregivers cope with the burden of care, the authors identified 27 treatments that could be synthetized as: support group, education, psycho-education, counseling, respite care, and multicomponent. Most of these interventions were individually effective, but with small effect sizes; nevertheless, the meta-analytic analysis showed that, collectively, these interventions yielded very limited overall effects on caregiver burden - except for multicomponent interventions, which more consistently reduced caregiver burden. The authors concluded that better interventions and more precise measures are needed to evaluate the effects of such interventions on caregiver burden. Given the highly emotional nature of the caregiver role and the inevitable interplay with personality factors, resulting in high a prevalence of depression, anxiety, and psychosomatic symptoms, it is reasonable to assume that psychologically-oriented interventions may be more reaching than informative methods based on support, education and counseling. Accordingly, the present set of data suggests that psychologically oriented interventions may be more effective than other types of intervention in the reduction of burden and depressive symptoms of family caregivers to AD patients. Yet, the superiority of this method has to be confirmed by additional controlled trials.

\section{Conclusion}

PGT and BAT were efficient in reducing caregiver burden in families of patients with mild- or moderate dementia due to AD. Both interventions yielded better estimates of quality of life. PGT additionally attenuated depressive symptoms. We understand that both interventions may yield complementary benefits to the standard clinical management of dementia due to $\mathrm{AD}$.

\section{Acknowledgements}

Financial support: Fundação de Amparo à Pesquisa de São Paulo (Fapesp Grant n 09/52825-8, Brazil), Associação Beneficente Alzira Denise Hertzog da Silva (ABADHS), JNK Empreendimentos e Incorporações.

\section{Conflict of interest}

None.

\section{References}

1. Elliott AF, Burgio LD, Decoster J. Enhancing caregiver health: findings from the resources for enhancing Alzheimer's caregiver health II intervention. J Am Geriatr Soc. 2010;58(1):30-7. 
2. Thompson CA, Spilsbury K, Hall J, Birks Y, Barnes C, Adamson J. Systematic review of information and support interventions for caregivers of people with dementia. BMC Geriatr. 2007;7:18.

3. Grossberg GT, Christensen DD, Griffith PA, Kerwin DR, Hunt G, Hall EJ. The art of sharing the diagnosis and management of Alzheimer's disease with patients and caregivers: recommendations of an expert consensus panel. Prim Care Companion J Clin Psychiatry. 2010;12(1):PCC.09cs00833.

4. Schulz R, McGinnis KA, Zhang S, Martire LM, Hebert RS, Beach SR, et al. Dementia patient suffering and caregiver depression. Alzheimer Dis Assoc Disord. 2008;22(2):170-6.

5. Austrom MG, Lu Y. Long term caregiving: helping families of persons with mild cognitive impairment cope. Curr Alzheimer Res. 2009;6(4):392-8.

6. Steadman PL, Tremont G, Davis JD. Premorbid relationship satisfaction and caregiver burden in dementia caregivers. J Geriatr Psychiatry Neurol. 2007;20(2):115-9.

7. Gaugler JE, Mittelman MS, Hepburn K, Newcomer R. Predictors of change in caregiver burden and depressive symptoms following nursing home admission. Psychol Aging. 2009;24(2):385-96.

8. Nichols LO, Chang C, Lummus A, Burns R, Martindale-Adams J, Graney MJ, et al.; Resources for Enhancing Alzheimer's Caregivers Health II Investigators. The cost-effectiveness of a behavior intervention with caregivers of patients with Alzheimer's disease. J Am Geriatr Soc. 2008;56(3):413-20.

9. Ryan KA, Weldon A, Huby NM, Persad C, Bhaumik AK, Heidebrink JL,et al. Caregiver support service needs for patients with mild cognitive impairment and Alzheimer disease. Alzheimer Dis Assoc Disord. 2010;24(2):171-6.

10. Hilgeman MM, Allen RS, DeCoster J, Burgio LD. Positive aspects of caregiving as a moderator of treatment outcome over 12 months. Psychol Aging. 2007;22(2):361-71.

11. Haley WE, Bergman EJ, Roth DL, McVie T, Gaugler JE, Mittelman MS. Long-term effects of bereavement and caregiver intervention on dementia caregiver depressive symptoms. Gerontologist. 2008;48(6):732-40.

12. Zarit S, Zarit J. The Memory and Behaviour Problems Checklist 1987R and the Burden Interview. Department of Individual and Family Studies, University Park. PA. 1990. In: Lu L, Wang L, Yang X, Feng Q. Zarit
Caregiver Burden Interview: development, reliability and validity of the Chinese version. Psychiatry Clin Neurosci. 2009;63:730-4.

13. Beck AT, Ward C, Mendelson M. Beck Depression Inventory (BDI). Arch Gen Psychiatry. 1961;4:561-71.

14. The World Health Organization quality of life assessment (WHOQOL) development and general psychometric properties. Soc Sci Med. 1998;46(12):1569-85.

15. Shields SA, Mallory MA, Simon A. The Body Awareness Questionnaire: reliability and validity. J Pers Assess. 1989;53:802-15.

16. Morris J. The Clinical Dementia Rating (CDR): current version and scoring rules. Neurology. 1993;43(11):2412-4.

17. Bittencourt MT. Consciência pelo movimento: o método Feldenkrais Jung \& Corpo. 1995;1:1.

18. Sandór P. Técnicas de relaxamento. São Paulo: Vetor Editora; 1974.

19. Kendall SA, Brolin-Magnusson K, Sören B, Gerdle B, Henriksson KG. A pilot study of body awareness programs in the treatment of fibromyalgia syndrome. Arthritis Care Res. 2000;13(5):304-11.

20. Maddali Bongi S, Di Felice C, Del Rosso A, Landi G, Maresca M, Giambalvo Dal Ben G, et al. Efficacy of the "body movement and perception" method in the treatment of fibromyalgia syndrome: an open pilot study. Clin Exp Rheumatol. 2011;29(6 Suppl 69):S12-8.

21. Farran CJ, Staffileno BA, Gilley DW, McCann JJ, Yan Li, Castro CM, et al. A A lifestyle physical activity intervention for caregivers of persons with Alzheimer's disease. Am J Alzheimers Dis Other Demen. 2008;23(2):132-42.

22. Kwok T, Wong B, Ip I, Chui K, Young D, Ho F. Telephone-delivered psychoeducational intervention for Hong Kong Chinese dementia caregivers: a single-blinded randomized controlled trial. Clin Interv Aging. 2013;8:1191-7.

23. Brown KW, Coogle CL, Wegelin J. A pilot randomized controlled trial of mindfulness-based stress reduction for caregivers of family members with dementia. Aging Ment Health. 2015:1-10.

24. Chen HM, Huang MF, Yeh YC, Huang WH, Chen CS. Effectiveness of coping strategies intervention on caregiver burden among caregivers of elderly patients with dementia. Psychogeriatrics. 2015;15(1):20-5.

25. Acton GJ, Kang J. Interventions to reduce the burden of caregiving for an adult with dementia: a meta-analysis. Res Nurs Health. 2001;24(5): $349-60$. 